\title{
Potential Virulence of Klebsiella Isolated from Enteral DietsVirulence of Klebsiella and Enteral Diets
}

\author{
Simone Cardoso Lisboa Pereira ${ }^{{ }^{*}}$ and Maria Cristina Danta Vanetti ${ }^{2}$ \\ ${ }^{1}$ Federal University of Minas Gerais, School of Nursing, Department of Nutrition, Belo Horizonte - Minas Gerais, Brazil \\ ${ }^{2}$ Federal University of Viçosa, Department of Microbiology, Viçosa - Minas Gerais, Brazil
}

\begin{abstract}
Objective: To evaluate the potential virulence of Klebsiella isolates from enteral diets in hospitals, in order to support nosocomial infection control measures, especially among critical patients.

Methods: Capsular phenotypic expression of the external membrane, production of aerobactin siderophore, quantity of capsular polysaccharide, hemolytic and phospholipase activity and resistance to antibiotics that are used therapeutically were investigated in 15 strains of $\mathrm{K}$. pneumoniae and six of K. oxytoca. These isolates were obtained from enteral diets in two public hospitals in the state of Minas Gerais, Brazil.

Results: The hypermucoviscous phenotype was observed in one of the K. pneumoniae isolates (6.7\%). Capsular serotypes of types $\mathrm{K} 1$ to $\mathrm{K} 6$ were seen to be present, of which four were $\mathrm{K} 5$ isolates of $\mathrm{K}$. pneumoniae and one was $\mathrm{K} 4$. Under the conditions of this study, no aerobactin production, hemolytic activity or lecithinase activity was observed. All the isolates presented resistance to the antibiotics amoxicillin and ampicillin, but they were sensitive to the antibiotics cefetamet, imipenem, cloranfenicol, gentamicin and sulfamethoxazole/trimethoprim. The K. pneumoniae isolates that originated from hospital B presented higher frequency of resistance to the antibiotics evaluated and multiple resistances to at least four antibiotics. The variations in the profile of resistance to antibiotics among the Klebsiella isolates made it possible to classify them into eight antibiotypes. No production of broad-spectrum $\beta$-lactamases was observed among the isolates.
\end{abstract}

Conclusion: The data obtained through this study favor the hypothesis that Klebsiella isolates from enteral diets are potential pathogens for nosocomial infections.

Keywords: Klebsiella; Enteral diets; Pathogenicity

\section{Introduction}

Bacteria of the genus Klebsiella frequently cause nosocomial infections and are associated with high morbidity and mortality [1]. In particular, K. pneumoniae, the species that is clinically most important, occurs in nosocomial infections such as those of the urinary tract and in cases of pneumonia and septicemia [1].

Given the severity of the worldwide situation regarding nosocomial infections, control efforts should be directed towards identifying the sources of contamination and the means of transmission, in order to implement preventive measures [2]. The gastrointestinal tract and the hands of hospital professionals are recognized sources of contamination by Klebsiella $[3,4]$ but, although this bacterium is frequently found in enteral diets, the involvement of these foods as a source of this opportunistic pathogen has been little described in the literature [5].

Recently, K. pneumoniae was identified as the nosocomial pathogen responsible for a large-scale outbreak, via the food chain. This was the first study to address the transmission of multiresistant Klebsiella through food in the hospital environment [6]. Moreover, according to Podschun et al. [7], K. pneumoniae isolates from other, nonclinical sources are also capable of expressing virulence factors, just like clinical isolates. Among the factors that contribute towards the pathogenicity of Klebsiella, the nature of the polysaccharide capsule of the external membrane, the production of siderophores and fimbrial adhesins and the quantity of capsular polysaccharide, as characterized by the mucoid appearance of the colonies, are considered to be the basis for this virulent process $[7,8]$.

Other factors have also been recognized and are considered to be intensifiers of the virulent potential of Klebsiella, such as hemolysin and phospholipase production and multiple resistance to antibiotics for therapeutic use [8]. It should be noted that the numbers of outbreaks of nosocomial infection caused by Klebsiella that is multiresistant to antibiotics, particularly extended-spectrum beta-lactamase (ESBL) producers, have been increasing over the last few years [9].

Considering that enteral diets have already been shown to be a potential cause of dissemination of Klebsiella in nosocomial environments; that nonclinical isolates may express virulence factors; that a recent study revealed that $K$. pneumoniae was the cause of a significant outbreak of nosocomial infection via the food chain; and that no study similar to the present study on enteral diets was found in the literature consulted; the objective of the present study was to evaluate the pathogenicity of Klebsiella strains isolated from modular enteral diets, by means of their phenotypic expression of virulence factors and from their profile of resistance to antibiotics.

\section{Methods}

\section{Control bacterial strains and origin of the isolates}

Strains of Escherichia coli were used as controls in the experiments to determine phenotypes of virulence factors, and these are described

*Corresonding author: Simone Cardoso Lisboa Pereira, Professor Federal University of Minas Gerais, School of Nursing, Department of Nutrition, Belo Horizonte-Minas Gerais, Brazil, Tel: 55313409 8028; E-mail: simoneclpereira@gmail.com

Received July 25, 2014; Accepted October 10, 2014; Published October 17 2014

Citation: Pereira SCL, Vanetti MCD (2014) Potential Virulence of Klebsiella Isolated from Enteral DietsVirulence of Klebsiella and Enteral Diets. J Food Process Technol 5: 383. doi:10.4172/2157-7110.1000383

Copyright: (c) 2014 Pereira SCL, et al. This is an open-access article distributed under the terms of the Creative Commons Attribution License, which permits unrestricted use, distribution, and reproduction in any medium, provided the original author and source are credited. 
in Table 1. The virulent potential of isolates of Klebsiella sp obtained from samples of modular diets used at two public hospitals in Brazil was evaluated and is presented in Table 2.

\section{Phenotypic determination of virulence factors}

All reagents used were analytical or HPLC grade, crystalline form of patulin (P1639, analytical reagent grade, Sigma-Aldrich Chemie GmbH Deisenhofen, Germany) and crystalline form ergosterol (E6510, analytical reagent grade, Sigma-Aldrich Chemie GmbH Deisenhofen, Germany) were used to prepare standard solutions. Also ultra-pure distilled water was used for chromatographic analysis.

\section{Mucoid appearance, presence of capsules and serotyping}

The mucoid appearance of the colonies was observed after incubation of the Klebsiella isolates in Brain Heart Infusion (BHI) agar, at $37^{\circ} \mathrm{C}$ for 48 hours. Individual colonies were tested regarding their capacity to form viscous chains. Formation of chains greater than $5 \mathrm{~mm}$ in length was indicative of a positive phenotype for hypermucoviscosity [10].

The presence of a capsule in the Klebsiella isolates was observed by viewing them under an optical microscope (Leica, model DMLS, Leica Microsystems, Germany) using an oil immersion lens, after preparing slides that were treated with Congo red. The unstained region surrounding the red central nucleus of the bacterial cells, on a blue background, indicated the presence of a capsule [10]. In addition, the isolates of K. pneumoniae were sent to the Enterobacteria Laboratory, Department of Bacteriology, Oswaldo Cruz Foundation, Rio de Janeiro, for serotyping evaluation regarding the capsular antigens $\mathrm{K} 1$ to $\mathrm{K} 6$, of the external membrane.

\section{Analysis of aerobactin production}

The aerobactin production was demonstrated using the LG 1522 strain of Escherichia coli as an indicator (Table 1) [11]. This strain was cultivated in M9 agar contained in Petri dishes, with the addition of 200 $\mu \mathrm{M}$ of 2,2 ' bipyridine (Sigma Chemical Co, St Louis, MO, USA). One colony of each Klebsiella isolate to be tested was inoculated into this agar, already containing the indicator strain. Aerobactin-producing strains were viewed as the halo of LG $1522 \mathrm{E}$. coli cell growth around the inoculated isolates. The positive control consisted of the E. coli strain LG 1315, and the negative control was the E. coli strain HB101 (Table 1).

\section{Phosphatidylcholine (PC-PLC) and hemolysin activity}

The PC-PLC activity was determined according to the methodology described by Coffey et al. [12]. The Klebsiella cultures were grown in $4 \mathrm{~mL}$ of $\mathrm{BHI}$ broth and were incubated at $37^{\circ} \mathrm{C}$ for 18 to 24 hours. Aliquots of $3 \mu \mathrm{L}$ of each culture were inoculated into the surface of BHI agar containing $2 \% \mathrm{NaCl}$ and $5 \%$ egg yolk emulsion, previously diluted in $0.85 \% \mathrm{NaCl}(1: 1, \mathrm{v} / \mathrm{v})$.

The plates were incubated at $37^{\circ} \mathrm{C}$ for 96 hours, in a Gas-Pak jar with an anaerobiosis generator (AnaeroGenTM, Oxoid, Basingstoke, England), and were observed to see whether a lecithin hydrolysis halo was present surrounding the colonies.

The hemolytic activity of the Klebsiella cultures was investigated using blood agar, as described by Kokosharov and Phetisova [13]. BHI plates (Oxoid, Basingstoke, England) containing 5\% defibrinated rabbit blood were prepared and small openings of $3 \mathrm{~mm}$ were drilled. These openings were used to inoculate $5 \mu \mathrm{L}$ of bacterial suspension, and the plates were then incubated at $37^{\circ} \mathrm{C}$ for 12 hours.

\section{Susceptibility to antibiotics}

The susceptibility of the Klebsiella isolates to the antimicrobial agents was determined through the disk diffusion method in MuellerHinton agar, in accordance with the guide from the National Committee for Clinical Laboratory Standards (NCCLS) [14]. The antibiotics used (Sensibiodisc-CECON, São Paulo, Brazil) were: nalidixic acid (NAL) $(30 \mu \mathrm{g})$; amikacin (AMI) $(30 \mu \mathrm{g})$; amoxicillin (AMO) $(10 \mu \mathrm{g})$; amoxicillin + clavulanic acid (AMC) $(30 \mu \mathrm{g} / 10 \mu \mathrm{g})$; ampicillin (AMP) $(10 \mu \mathrm{g})$; chloramphenicol (CLO) $(30 \mu \mathrm{g})$; gentamicin (GEN) $(10 \mu \mathrm{g})$; imipenem (IMP) $(30 \mu \mathrm{g})$; kanamycin $(\mathrm{KN})(30 \mu \mathrm{g})$; neomycin (NO) $(30 \mu \mathrm{g})$; tetracycline (TET) $(30 \mu \mathrm{g})$; ticarcillin + clavulanic acid (TIC) $(75 \mu \mathrm{g} / 10 \mu \mathrm{g})$; and trimethoprim + sulfamethoxazole (SUT) $(1.25 / 23.75$ $\mu \mathrm{g})$. To diagnose strains producing ESBL, screening was performed using the following antibiotics: cefotaxime (CTX) $(30 \mu \mathrm{g})$; ceftazidime (CAZ) $(30 \mu \mathrm{g})$; cefetamet (CEF) $(10 \mu \mathrm{g})$; cefalotin (CFL) $(30 \mu \mathrm{g})$; and aztreonam (ATM) $(30 \mu \mathrm{g})$, in accordance with the recommendations of the NCCLS [14]. After incubation at $35^{\circ} \mathrm{C}$ for 16 to 18 hours, isolates that, in accordance with the antibiotics tested, presented the following growth inhibition halos were considered to be suspected ESBL producers: cefetamet $\leq 22 \mathrm{~mm}$; ceftazidime $\leq 22 \mathrm{~mm}$; aztreonam $\leq 27$ $\mathrm{mm}$; cefotaxime $\leq 27 \mathrm{~mm}$; and cefalotin $\leq 25 \mathrm{~mm}$. As a confirmatory test, the E test was performed (AB BIODISK, Solna, Sweden), using the antibiotics ceftazidime and cefotaxime as the substrate. Reduction of the minimum inhibitory concentration (MIC) of the antimicrobial agent in association with beta-lactamase inhibitor, in comparison with the MIC of the antimicrobial agent beta-lactamase alone (ceftazidime or cefotaxime), was indicative of ESBL-producing strains.

\section{Results}

The majority of the Klebsiella isolates obtained from the modular enteral diets formed colonies with low intensity of mucoid appearance in BHI agar. Four isolates (26.7\%) of K. pneumoniae presented moderate mucoid appearance and only the isolate U4 (6.7\%) presented a phenotype of hypermucoviscosity. The presence of a capsule in the isolate $\mathrm{U} 4$, and also in the isolates with colonies of moderate mucoid appearance was confirmed through observing the preparations under the microscope. In the isolates that presented colonies with low mucoid appearance, it was not possible to observe any capsule through the staining used. In addition, five isolates with the presence of capsule antigens between $\mathrm{K} 1$ and $\mathrm{K} 6$ were identified (Table 2).

Under the experimental conditions used, the Klebsiella isolates from enteral diets did not present any aerobactin production, hemolytic activity or lecithinase activity.

All the 21 Klebsiella isolates from enteral diets presented resistance to the antibiotics amoxicillin and ampicillin (Table 3). However, when amoxicillin was used in association with clavulanic acid, which is a beta-lactamase inhibitor, six isolates (28.6\%) were resistant (Table 3). This behavior was also observed in relation to the association between ticarcillin and clavulanic acid, but less frequently (Table 3).

The Klebsiella isolates were sensitive to antibiotics of the betalactamic group: cefetamet, which is a third-generation cephalosporin; and imipenem, which is a carbapenem (Table 3). These isolates were also inhibited by antibiotics of different groups, such as: chloramphenicol (a quinolone); gentamicin (an aminoglycoside); and sulfatrim (an association between trimethoprim and sulfamethoxazole).

It was observed that the isolates of $K$. pneumoniae from enteral diets that originated from hospital B presented greater frequency of isolates resistant to the antibiotics evaluated and multiple resistance to at least 
Citation: Pereira SCL, Vanetti MCD (2014) Potential Virulence of Klebsiella Isolated from Enteral DietsVirulence of Klebsiella and Enteral Diets. J Food Process Technol 5: 383. doi:10.4172/2157-7110.1000383

Page 3 of 6

\begin{tabular}{|c|c|c|c|}
\hline Sample & Strains & Relevant properties or characteristics & Source or reference \\
\hline 1 & E. coli LG1315 & ara entA lac leu mtL proC rpsL supE thi tonA trpE Xyl, controle negativo & WILLIAMS (1979) \\
\hline 2 & E. coli LG1522 & Ara azi fepA lac leu mtL proC rpsL supE tonA tsx thi col-V-K30iuc, controle positivo & WILLIAMS (1979) \\
\hline 3 & E. coli F205 & Produtora de cloacina DF13 & WILLIAMS (1979) \\
\hline
\end{tabular}

Table 1: Strains of Escherichia coli used as controls in this study

\begin{tabular}{|c|c|c|c|c|c|c|}
\hline \multirow[b]{2}{*}{$\begin{array}{l}\text { Isolate/ } \\
\text { Origin }\end{array}$} & \multirow[b]{2}{*}{ Espécie } & \multicolumn{5}{|c|}{ Virulence phenotypes } \\
\hline & & $\begin{array}{l}\text { Aspect } \\
\text { mucoid }\end{array}$ & $\begin{array}{l}\text { Capsular } \\
\text { serotype }\end{array}$ & $\begin{array}{l}\text { Aerobactin } \\
\text { production }\end{array}$ & Hemolytic & Lecithinase activity \\
\hline $\mathrm{P} 1 / \mathrm{A}$ & K. oxytoca & Absent & $-\mathrm{a}^{\mathrm{a}}$ & Negative & Negative & Negative \\
\hline $\mathrm{P} 2 / \mathrm{A}$ & K. oxytoca & Absent & - & Negative & Negative & Negative \\
\hline $\mathrm{P} 3 / \mathrm{A}$ & K. oxytoca & Absent & - & Negative & Negative & Negative \\
\hline $\mathrm{P} 4 / \mathrm{A}$ & K. oxytoca & Absent & - & Negative & Negative & Negative \\
\hline $\mathrm{P} 5 / \mathrm{A}$ & K. oxytoca & Absent & - & Negative & Negative & Negative \\
\hline P6/A & K. pneumoniae & Weak & $\mathrm{ND}^{\mathrm{b}}$ & Negative & Negative & Negative \\
\hline P7/A & K. pneumoniae & Weak & ND & Negative & Negative & Negative \\
\hline $\mathrm{P} 8 / \mathrm{A}$ & K. pneumoniae & Weak & ND & Negative & Negative & Negative \\
\hline $\mathrm{P} 9 / \mathrm{A}$ & K. oxytoca & Absent & - & Negative & Negative & Negative \\
\hline $\mathrm{P} 11 / \mathrm{A}$ & K. pneumoniae & Weak & ND & Negative & Negative & Negative \\
\hline $\mathrm{P} 13 / \mathrm{A}$ & K. pneumoniae & Moderate & K5 & Negative & Negative & Negative \\
\hline P14/A & K. pneumoniae & Weak & ND & Negative & Negative & Negative \\
\hline P15/A & K. pneumoniae & Weak & ND & Negative & Negative & Negative \\
\hline P17/A & K. pneumoniae & Weak & ND & Negative & Negative & Negative \\
\hline U1/B & K. pneumoniae & Moderate & K5 & Negative & Negative & Negative \\
\hline U2/B & K. pneumoniae & Weak & ND & Negative & Negative & Negative \\
\hline U3/B & K. pneumoniae & Moderate & ND & Negative & Negative & Negative \\
\hline U4/B & K. pneumoniae & Intense & K5 & Negative & Negative & Negative \\
\hline U5/B & K. pneumoniae & Weak & K4 & Negative & Negative & Negative \\
\hline U7/B & K. pneumoniae & Absent & ND & Negative & Negative & Negative \\
\hline U8/B & K. pneumoniae & Moderate & K5 & Negative & Negative & Negative \\
\hline
\end{tabular}

$A=$ Hospital $A$

$B=$ Hospital $B$

$\mathrm{ND}=$ not serotyped

$\mathrm{b}=$ undetermined serotype, serotype $\mathrm{K} 6$ up

Table 2: Virulence phenotypes of Klebsiella isolates from modular enteral diets, originating from two public hospitals

\begin{tabular}{|c|c|c|c|c|}
\hline \multirow[b]{2}{*}{ Antibiotics } & \multicolumn{4}{|c|}{ Species/Hospital N (\%) } \\
\hline & K. pneumoniae /A & K. oxytoca /A & K. pneumoniae/B & Total \\
\hline $\begin{array}{c}\text { Amoxicillin }+ \text { Clavulanic Acid } \\
\text {-AMC }\end{array}$ & $2(25,0)$ & $0(0,0)$ & $4(57,1)$ & $6(28,6)$ \\
\hline Nalidixic acid-NAL & $2(25,0)$ & $0(0,0)$ & $6(85,7)$ & $8(38,1)$ \\
\hline Amikacin-AMI & $1(12,5)$ & $0(0,0)$ & $0(0,0)$ & $1(4,8)$ \\
\hline Amoxicillin-AMO & $8(100,0)$ & $6(100,0)$ & $7(100,0)$ & $21(100,0)$ \\
\hline Ampicillin -AMP & $8(100,0)$ & $6(100,0)$ & $7(100,0)$ & $21(100,0)$ \\
\hline Aztreonam-ATM & $2(25,0)$ & $0(0,0)$ & $6(85,7)$ & $8(38,1)$ \\
\hline Cephalothin-CFL & $2(25,0)$ & $0(0,0)$ & $5(71,4)$ & $7(33,3)$ \\
\hline Ceftamet-CEF & $0(0,0)$ & $0(0,0)$ & $0(0,0)$ & $0(0,0)$ \\
\hline Cefotaxime CTX- & $2(25,0)$ & $0(0,0)$ & $5(71,4)$ & $7(33,3)$ \\
\hline Ceftazidime-CAZ & $0(0,0)$ & $0(0,0)$ & $1(14,3)$ & $1(4,8)$ \\
\hline Chloramphenicol-CLO & $0(0,0)$ & $0(0,0)$ & $0(0,0)$ & $0(0,0)$ \\
\hline Gentamicin-GEN & $0(0,0)$ & $0(0,0)$ & $0(0,0)$ & $0(0,0)$ \\
\hline Imipenem-IPM & $0(0,0)$ & $0(0,0)$ & $0(0,0)$ & $0(0,0)$ \\
\hline Kanamycin-KN & $3(37,5)$ & $0(0,0)$ & $0(0,0)$ & $3(14,3)$ \\
\hline Neomycin-NO & $2(25,0)$ & $0(0,0)$ & $1(14,3)$ & $3(14,3)$ \\
\hline Sulfaxotrim-SUT & $0(0,0)$ & $0(0,0)$ & $0(0,0)$ & $0(0,0)$ \\
\hline Tetracycline-TET & $2(25,0)$ & $0(0,0)$ & $3(42,9)$ & $5(23,8)$ \\
\hline Ticarcillin $+\underset{\text { TIC }}{\text { clavulanic acid- }}$ & $1(12,5)$ & $0(0,0)$ & $0(0,0)$ & $1(4,8)$ \\
\hline
\end{tabular}

Table 3: Resistance profile shown by Klebsiella isolates to antimicrobial agents for therapeutic use in the hospitals studied 
four antibiotics was also more frequent in that hospital (Table 3).

The variation in the profile of resistance to antibiotics between the Klebsiella isolates made it possible to classify them into the antibiotypes described in Table 4.

ESBL production was not observed among the isolates of $K$. pneumoniae and K. oxytoca. This observation was possible because no reduction in the MIC of the antimicrobial agent in association with beta-lactamase inhibitor, in comparison with the MIC of the betalactamic antimicrobial agent alone (ceftazidime or cefotaxime) was seen.

The resistance to the antibiotics used as the substrate for detecting ESBL-producing strains ranged from 0 to $38.1 \%$ (Table 3). The highest frequencies of resistance were recorded for the beta-lactamic agents aztreonam and cefotaxime, with rates of 38.1 and $33.3 \%$ respectively, while the lowest frequencies of resistance were recorded for ceftazidime and cefetamet: 4.8 and $0 \%$ respectively (Table 3 ).

\section{Discussion}

In this study, the phenotype of hypermucoviscosity presented low frequency among the colonies formed by the isolates of K. pneumoniae (6.7\%). Vernet et al. [15] also observed that this appearance presented low frequency of occurrence, given that out of 241 clinical isolates of $K$. pneumoniae, only $7.0 \%$ presented this characteristic. In a study with greater coverage [16], with 455 clinical isolates of K. pneumoniae involved in consecutive cases of bacteremia, in seven countries, it was found that in two of these countries, the phenotypic frequency of hypermucoviscosity was $94 \%$ in isolates that caused pneumonia and $100 \%$ in isolates that caused invasive syndrome. However, in the other countries, this frequency was on average $2 \%$, i.e. lower than the frequency found in the present study.

The mucoid appearance of colonies is related to production of the capsule, which is a virulence factor that is essential for Klebsiella to be able to overcome the host's immune system [17]. Furthermore, a recent study implicated a role for the genes magA and $\mathrm{rmpA}$, as determinants of the phenotype of hypermucoviscosity, in the pathogenesis of the $\mathrm{K} 1$ capsular serotype of K. pneumoniae, which is involved in bacteremia [18]. The gene rmpA, which codes for a positive regulator for extracapsular mucopolysaccharide expression [16], was also identified in two (2/54) clinical isolates out of a total of five isolates with the phenotype of hypermucoviscosity, although they did not present K1-K6 serotyping [8]. The phenotype of hypermucoviscosity is seen predominantly in strains with the serotypes K1 and K2, which are clinical isolates of invasive diseases. This would explain the low rates of hypermucoviscosity among the isolates of the present study [8].
Presence of a capsule is an important virulence factor regarding the severity of the infection. A capsule is generally present in clinical strains, and 78 capsule types have been identified in K. pneumoniae. However, determination of capsule types is often difficult, because of the limitations of traditional serotyping [19]. In the present study, it was found that four isolates had the serotype $\mathrm{K} 5$ and one had the serotype $\mathrm{K} 4$, out of a possible range of six main serotypes for this microbial species (K1-K6). Among the 78 distinct capsule antigens of $K$. pneumoniae, only some of them, such as $\mathrm{K} 1, \mathrm{~K} 2$ and $\mathrm{K} 5$, are considered to be highly virulent and these are frequently associated with severe infections in humans and animals. One of these types of antigen was found in four of the isolates of this study.

None of the isolates of this study presented aerobactin production. According to several authors, Klebsiella isolates with phenotypes positive for aerobactin are rare, independent of the species or source of isolation $[7,8,15]$.

Aerobactin is one of the siderophores (extracellular ferric chelating agents) that are secreted by bacterial cells in order to eliminate $\mathrm{Fe} 3+$, from iron-binding proteins in the host. These have a critical role in bacterial virulence. Based on the results found here and information in the literature consulted, Klebsiella may be included in the group of enterobacteria that present low aerobactin production rates (less than $20 \%)$.

Differences in the research relation between industrial diets and modulated diets make them difficult to compare, evidence seems strong enough to take into account the intake the Degradation of Food and the Struggle for Healthy Eating which transformation of food from essential nutrients into edible commodities that far too often fail to nourish us. Modulated diets diets consisted of a control, nutritionally balanced diet, this same diet supplemented with $10 \%$ and a diet that was high in fat and carbohydrate and low in protein.

In this regard, considering that the bioavailability of iron in the host is extremely low (10-18 M); that iron is essential for microbial growth; and that the incidence of the phenotype of aerobactin is low among Klebsiella isolates, the question of which mechanisms might ensure an iron supply in the host for this bacterium is raised.

It has been shown that enterobactin, another ferric chelating agent, is present in the majority of clinical isolates of $K$. pneumonia $[8,11]$. The role of enterobactin in Klebsiella virulence is still uncertain, but expression of the enterobactin gene seems to be activated during the infection. A recent study found that, genotypically, enterobactin was present in clinical isolates of noninvasive infections (bacteremia) [8].

The absence of hemolytic activity under the conditions of this

\begin{tabular}{|l|l|l|}
\hline Antibiotype & Isolates ${ }^{*}$ & Antibiotic resistance: \\
\hline I & P1, P2, P3, P4, P5, P6, P7, P8, P9, P11, P13 e U5 & AMO e AMP \\
\hline II & U3 & NAL, AMO, AMP e ATM \\
\hline III & P15 & AMO, AMP,ATM, KN e TET \\
\hline IV & U2 & AMC, NAL, AMO, AMP, ATM, CFL e CTX \\
\hline V & U4 & NAL, AMO, AMP, ATM, CFL, CTX e TET \\
\hline VI & P17 & AMC, NAL, AMO, AMP, CFL, CTX, KN e NO \\
\hline VII & U1 & AMC, NAL, AMO, AMP, ATM, CFL, CTX e CAZ \\
\hline VIII & U7 & AMC, NAL, AMO, AMP, ATM, CFL, CTX, TET \\
\hline IX & U8 & AMC, NAL, AMO, AMP, ATM, CFL, CTX, NO e TET \\
\hline X & P14 & AMC, NAL, AMI, AMO, AMP, ATM, CFL, CTX, KN, NO, TET e TIC \\
\hline
\end{tabular}

* The isolates are specified in Table 2 and the antibiotics are described in Table 3

Serotyping not determined; serotypes higher than K6

Table 4: Determination of the antibiotypes of the Klebsiella isolates from enteral diets 
study may be related to a lack of activators for expressing the genes that code for this enzyme, which are often not reproducible under in vitro conditions [20]. This virulence factor was first detected by ALBESA in 1980, in a test conducted on blood agar containing rabbit erythrocytes. Since then, this research group has been studying this cytolysin, and its specificity for rabbit erythrocytes has always been confirmed. Hemolysin is a pore-forming toxin that makes nutrients available, such as the iron of hemoglobin. In a recent study, none of the 54 clinical isolates presented hemolysis or presence of the genes hlyA and cnf-1, which code for this activity. This is consistent with the lack of studies reporting genotypic and phenotypic presence of hemolysis in Klebsiella, even though hemolytic activity has been described $[20,21]$.

Lecithinase or PC-PLC activity was absent from all the isolates under the conditions in which this was determined. This result is concordant with that of Singh et al. [22] who reported that lecithinase activity was rare in Klebsiella isolates, given that only $2.4 \%$ of their 168 clinical isolates of $K$. pneumoniae presented this activity. Furthermore, they found that this activity was absent among the 11 isolates of $K$. oxytoca. Their study was the first report on lecithinase activity in Klebsiella isolates, and the role of this virulence factor in the pathogenicity of this genus of bacteria has not yet been fully clarified.

Among the antibiotics used in the present study, it was seen that there was high prevalence of resistance to ampicillin and amoxicillin. This observation regarding the antibiotic ampicillin was also made in a study on clinical isolates of K. pneumonia [23] and in a study on isolates of this enterobacterium in expressed human breastmilk [24].

Another point observed was that there was greater prevalence of resistance to antibiotics among the isolates from hospital B. According to Davies [23], the prevalence of resistance to antibiotics may vary between different hospitals and may be related to local epidemiological factors such as control over the use of antibiotics and preventive measures applied by hospital infection committees, in relation to the problem of multiresistant strains.

No correlation between high incidence of resistance to the antibiotic cefotaxime and ESBL production was observed. This is an important clinical result, even though the isolates presented multiple resistance to up to 12 antibiotics.

The condition of multiresistance observed in the present study confirms the assertion that bacterial resistance to antimicrobial agents is a serious public health problem. This is the reality within which control initiatives have been proposed. In Brazil in 2010, a resolution making provisions regarding controls over medications based on substances classified as antimicrobial agents for use under medical prescription singly or in combinations was instituted, such that dispensing of these substance could only be done through a specially controlled prescription [25].

Moreover, the observation of multiple resistance to antibiotics in Klebsiella isolates has significant clinical importance. This is because enteral diets are especially prescribed for patients who are highly susceptible to nosocomial infection and who are concentrated in intensive care units. These are patients who are subject to the intrinsic risks relating to complex underlying diseases, nutritional vulnerability, extreme ages (premature infants and elderly people) and effects of immunosuppression or immunodepression drugs, in addition to extended-spectrum antimicrobial agents. In addition, there are extrinsic risks such as prolonged stay in the hospital environment, subjection to routines of invasive procedures and quality that is not guaranteed for all hospital services, given that this is a complex organization. In this context, the risk to health becomes more severe, given that these situations may be sources of resistance genes for pathogenic microorganisms, particularly those that are toxic-infectious and infectious, since the intestinal tract constitutes an appropriate niche for transferring genes relating to resistance [26].

From results surveyed in the literature, it was seen that some authors have suggested that transfers of plasmids with the capacity to carry more than one associated resistance gene facilitate horizontal gene transmission of different resistance mechanisms between bacteria, thus further worsening the problem of multiple resistance to antibiotics in the hospital environment [23,27].

These transfers are probably more effective when the hosts are simultaneously subjected to selective pressure from an antimicrobial substance to which the microorganisms involved are resistant. From this point of view, hospitals are environments where drug-resistant microorganisms and others that determine such resistance are more likely to be present. Thus, horizontal gene transfer has a greater chance of occurring and the consequences, in terms of therapeutic failure, may be more severe $[28,29]$.

Occurrences of chromosomal mutations alone do not explain the rapid acquisition of gene resistance to antibiotics among bacteria. It has been established that plasmids are particularly important in the evolution of antibiotic-resistant bacteria. It has also been suggested that plasmid transfers carrying resistance genes facilitates horizontal gene transmission between bacteria $[23,27]$. Foods seem to be an effective source for human acquisition of resistant bacteria and gene resistance to drugs, but the extent and real consequences of this exposure have not yet been sufficiently investigated [30,31].

\section{Final Remarks}

The data obtained in this study favor the hypothesis that Klebsiella isolates from enteral diets are potential pathogens for nosocomial infections, especially for critical patients. This is because, among the isolates in this study, capsular serotypes that are only identified in severe cases of nosocomial infections were observed. These serotypes include $\mathrm{K} 5$ and $\mathrm{K} 4$, which are among the serotypes of clinical importance ( $\mathrm{K} 1$ to $\mathrm{K} 6$ ). In addition, presence of the phenotype of hypermucoviscosity was observed: this intensifies the invasion process in cases of isolates of one of these important capsular serotypes. This profile of virulence, which can be considered to partially form the basis for infection by Klebsiella, now represents a risk to microbiological security. This is especially so in situations of propagation in intensive care units, where the intrinsic and extrinsic risks favor conditions of nosocomial infection. Furthermore, the synergy that may exist between these virulence factors can be highlighted: this may especially occur in combination with multiple resistance to antibiotics for therapeutic use, as also seen in the present study. Thus, there is a need to continue this study in order to evaluate the pathogenicity of these isolates under in vivo conditions.

In addition, through these results, it can be seen that control measures against nosocomial infections are important in handling enteral diets. Through comprehension of this reality, the need for participation by all sectors of the hospital in the process of controlling this situation, regarding individual and collective practices, is corroborated. It can furthermore be emphasized that rigorous and systematic monitoring of the risks of food origin has to be applied to the hospital environment. Good food handling practices are effective strategies for controlling the dissemination of these and other bacteria in the hospital environment. 
Citation: Pereira SCL, Vanetti MCD (2014) Potential Virulence of Klebsiella Isolated from Enteral DietsVirulence of Klebsiella and Enteral Diets. J Food Process Technol 5: 383. doi:10.4172/2157-7110.1000383

In this regard, the present study adds knowledge regarding the nosocomial infections caused by Klebsiella and emphasizes the importance of measures relating to outcomes associated with the nutrition sector, with the aim of strengthening the interfaces between infection control and preventive actions and between these controls and promotion of quality within healthcare services overall. The present study also endorses the need to direct healthcare funding policies towards implementation of risk control and prevention measures in strategic sectors of the healthcare services. There is a need to reflect on all the possible strategies that might contribute towards changing the current panorama of nosocomial infections, with investments in research and updates. It can be emphasized that dissemination of data analyses like the present study and other recent studies should be done routinely for all the professionals involved in care, as well as within the institution's administration.

\section{References}

1. Lin YT, Wang FD, Chan YJ, Fu YC, Lin CPF, et al (2014) Clinical and microbiological characteristics of tigecycline non-susceptible Klebsiella pneumoniae bacteremia in Taiwan. BMC Infect Dis 14:1.

2. WHO World Health Organization (2011) Core components for infection prevention and control programs. Assessment tools for IPC programmes. Geneve, World Health Organization (WHO). Prevention of hospital-acquired infections.

3. Ekrami AR, Kayedani A, Jahangir M, Kalantar E, Jalali M (2011) Isolation of common aerobic bacterial pathogens from the environment of seven hospitals, Ahvaz, Iran. Jundishapur J Microbiol 4: 75-82.

4. Westerbeek EAM, Berg AVD, Lafeber HN, Fetter WPF, Elburg RMV (2011) The effect of enteral supplementation of a prebiotic mixture of non-human milk galacto-fructo- and acidic oligosaccharides on intestinal permeability in preterm infants. Br J Nutr 105: 268-274.

5. Hurrell E, Kucerova E, Loughlin M, Caubilla-Barron J, Hilton A, et al. (2009) Neonatal enteral feeding tubes as loci for colonisation by members of the Enterobacteriaceae. BMC Infect Dis 9: 146.

6. Calbo E, Freixas N, Xercavins M, Riera M, Nicola's C, et al. (2011) Foodborne nosocomial outbreak of SHV1 and CTX-M-15-producing Klebsiella pneumoniae: epidemiology and control. Clin Infect Dis 52: 743-749.

7. Podschun R, Pietsch S, Holler C, Ullmann U (2001) Incidence of Klebsiella species in surface waters and their expression of virulence factors. Appl Environm Microbiol 67: 3325-3327.

8. Fertas AR, Messai Y, Alouache S, Bakour R (2013) Virulence profiles and antibiotic susceptibility patterns of Klebsiella pneumonia strains isolated from different clinical specimens. Pathologie Biologie 61: 209-216.

9. Viale P, Giannella M, Lewis R, Trecarichi EM, Petrosillo N, et al. (2013) Predictors of mortality in multidrug-resistant Klebsiella pneumoniae bloodstream infections. Expert Review of Anti-infective Therapy 1: 1053-1063.

10. Wiskur BJ, Hunt J, Callegan MC (2008) Hypermucoviscosity as a virulence factor in experimental Klebsiella pneumoniae endophthalmitis. Invest Ophthalmol Vis Sci 49: 4931-4938.

11. Podschun R, Sievers D, Foscher A, Ullmann U (1993) Serotypes, hemagglutinins, siderophore synthesis, and serum resistance of Klebsiella isolates causing human urinary tract infections. J Infect Dis 168:1415-1421.

12. Coffey A, Rombouts FM, Abee T (1996) Influence of environmental parameters on phosphatidylcholina phospholipase $\mathrm{C}$ production in Listeria monocytogenes from other Listeria species. Appl Environ Microbiol 62: 1252-1256.

13. Kokosharov T, Phetisova K (2002) Hemolysins and aerobactin in Salmonella Gallinarum strains isolated from poultry. Rev Med Vet 153: 411-414.

14. NCCLS National Committe For Clinical Laboratory Standards (2003) Performance Standards for Antimicrobial Disk Susceptibility Tests; Approved Standard. (8thedn). NCCLS document M2-A8 (ISBN 1-56238-485-6). NCCLS, 940 West Valley Road, Suite 1400, Wayne, Pennsylvania 19087-1898 USA.

15. Vernet V, Madoulet V, Chippaux V, Philippon A (1992) Incidence of two virulence factors (aerobactin and mucoid phenotype) among 190 clinical isolates of Klebsiella pneumoniae producing extended-spectrum beta-lactamase. FEMS Microbiol Lett 75: 1-5.
16. Yu VL, Hansen DS, Ko WC, Sagnimeni A, Klugman KP, et al. (2007) Virulence characteristics of Klebsiella and clinical manifestations of K. pneumoniae bloodstream infections. Emerg Infect Dis 13: 986-993.

17. Evrard B, Balestrino D, Dosgilbert A, Bouya-Gachancard J, Charbonnel N, et al. (2010) Roles of Capsule and Lipopolysaccharide O Antigen in Interactions of Human Monocyte-Derived Dendritic Cells and Klebsiella pneumonia. Infect Immun 78: 210-219.

18. Cheng KC, Lee MF, Chuang YC, Yu WL (2013) First description of lung abscess caused by ST23 clone capsule genotype K1 Klebsiella pneumonia. J Formos Med Assoc 613: 293-293.

19. Pan YJ, Lin TL, Chen YH, Hsu CR, Hsieh PF, et al. (2013) Capsular Types of Klebsiella pneumoniae Revisited by wzc Sequencing. PLoS ONE 8: e80670.

20. Albesa I (1989) Klebsiella pneumoniae haemolysin adsorption to red blood cells. J Appl Bacteriol 67: 263-266.

21. Gundogan N, Citak S, Yalcin E (2011) Virulence properties of extendedspectrum beta-lactamase-producing Klebsiella species in meat samples. J Food Prot 74: 559-564.

22. Singh BR, Sharma VD, Chandra R (1999) Detection, prevalence, purification and characterization of lecithinase de Klebsiella pneumoniae. Indian J Exp Biol 7: $925-932$.

23. Davies J, Davies D (2010) Origins and Evolution of Antibiotic Resistance. Microbiology and Molecular Biology Reviews 74: 417-433.

24. Novak FR, Almeida JAG, Asensi MD, Moraes BA, Rodrigues DP (2001) Resistência antimicrobiana de coliformes isolados de leite humano ordenhado. Cad Saúde Pública 17: 713-717.

25. Brasil (2011) Ministério da Saúde. Agência Nacional de Vigilância Sanitária - ANVISA. Resolução de Diretoria Colegiada (RDC) n²0, de 5 de maio de.

26. Andremont A (2003) Commensal Flora May Play Key Role in Spreading Antibiotic Resistance. ASM news 69: 601-607.

27. Herrera-Leon S, González-Sanz R, Herrera-Leon L, Echeita MA (2011) Characterization of multidrug-resistant Enterobacteriaceae carrying plasmidmediated quinolone resistance mechanisms in Spain. Antimicrob Chemotherv 66: 287-290.

28. Alekshun MN, Levy BS (2007) Molecular mechanisms of antibacterial multidrug resistance. Cell 128: 1037-1050.

29. Evans HL, SN Lefrak, Lyman J (2007) Cost of Gram-negative resistance. Critical Care Medicine 1: 89-95.

30. Machado E, Coque TM, Canton R, Sousa JC, Peixe L (2008) Antibiotic resistance integrons and extended-spectrum $\beta$-lactamases among Enterobacteriaceae isolates recovered from chickens and swine in Portugal. $J$ Antimicrob Chemother 62: 296-302.

31. Hilary AM, Samuel W, Walker I, Sadler DA, Evans K (2011) Age-Related Biomarkers Can be Modulated by Diet in the Rat. FNS 2: 884-890. 